\title{
Measured Total Cross Sections of Slow Neutrons Scattered by Solid Deuterium and Implications for Ultracold Neutron Sources
}

\author{
F. Atchison, B. Blau, B. van den Brandt, T. Bryś, ${ }^{*}$ M. Daum, P. Fierlinger, ${ }^{\dagger}$ P. Hautle, R. Henneck, S. Heule, ${ }^{\dagger}$ K. Kirch, ${ }^{\dagger}$ \\ J. Kohlbrecher, G. Kühne, J. A. Konter, A. Pichlmaier, and A. Wokaun* \\ Paul Scherrer Institut (PSI), CH-5232 Villigen PSI, Switzerland ${ }^{\S}$ \\ K. Bodek, M. Kasprzak," and M. Kuźniak ${ }^{\text {II }}$ \\ Institute of Physics, Jagiellonian University, 30059 Krakow, Poland \\ P. Geltenbort \\ Institut Laue-Langevin (ILL), 38042 Grenoble, France
}

J. Zmeskal

Stefan Meyer Institut für subatomare Physik, Austrian Academy of Sciences, 1090 Vienna, Austria

(Received 15 July 2005; published 25 October 2005)

\begin{abstract}
The total scattering cross sections for slow neutrons with energies in the range $100 \mathrm{neV}$ to $3 \mathrm{meV}$ for solid ortho- ${ }^{2} \mathrm{H}_{2}$ at 18 and $5 \mathrm{~K}$, frozen from the liquid, have been measured. The $18 \mathrm{~K}$ cross sections are found to be in excellent agreement with theoretical expectations and for ultracold neutrons dominated by thermal up scattering. At $5 \mathrm{~K}$ the total scattering cross sections are found to be dominated by the crystal defects originating in temperature induced stress but not deteriorated by temperature cycles between 5 and $10 \mathrm{~K}$.
\end{abstract}

DOI: 10.1103/PhysRevLett.95.182502

PACS numbers: 28.20.-v, 28.20.Cz, 61.12.Ex

Neutrons are termed "ultracold" when they are slow enough to be confined in traps. The traps themselves may be formed by a combination of suitable material walls, magnetic fields, and gravitation. Stored ultracold neutrons (UCN) behave in many respects like an ideal gas at a temperature in the milli-Kelvin region and may be observed for very long times, in principle only limited by the lifetime due to $\beta$ decay. UCN are predominantly used for fundamental physics experiments, such as searches for an electric dipole moment of the neutron or measurements of the neutron lifetime; see [1,2]. At the present, most of these experiments, as well as long dreamed about applications of UCN, e.g., in the study of surfaces or macromolecules, are limited by the UCN intensities available being too low. Improvements in UCN source strength are urgently needed.

The potential of solid deuterium as a good cold neutron moderator and UCN converter was recognized more than 20 years ago [3-5] and has been demonstrated in various test experiments [6-9] only in the past few years. Large gain factors in comparison with gaseous and liquid ${ }^{2} \mathrm{H}_{2}$ [3,6], reasonably long UCN lifetimes [7], and a large absolute production rate [8] were measured. As a result it appears feasible to build high intensity UCN sources using solid ${ }^{2} \mathrm{H}_{2}$ [10,11]. It was pointed out in [12,13] that high UCN densities should be obtained by using solid deuterium in combination with a pulsed neutron source at low repetition rate. The UCN source prototype realized in $[7,8]$ and the UCN source under construction at PSI [11] rely on this concept. Recently the UCN production cross section from cold neutrons has been measured [9] and found to be in good agreement with theoretical expectations [5]. Besides knowing the UCN production cross section and the UCN lifetime it is also important to know the scattering cross section for UCN inside solid deuterium, as this determines the mean free path for extraction of UCN from the solid deuterium converter to connected UCN storage volumes and experiments. For a pulsed UCN source the deuterium temperature will increase during the production pulse and it is important to check on whether this causes deterioration of the UCN production.

We report here on measurements of neutron transmission through solid deuterium targets prepared by freezing from the liquid phase. The crystals were grown slowly (over about $12 \mathrm{~h}$ ) at a temperature close to the triple point (see [14]) and then either held at $18 \mathrm{~K}$ or further cooled slowly to $5 \mathrm{~K}$. The $5 \mathrm{~K}$ crystals were also subjected to thermal cycles between 5-10 K and 5-18 K, respectively. We used the same equipment and procedures for preparation of solid deuterium as used in earlier studies [14]. The cryogenic target cell is the same one used in our recent measurements of scattering cross sections for gaseous and liquid deuterium [15]. Details about the experimental setup and data treatment procedures are given in $[16,17]$.

The measurements have been performed using cold neutrons $(\mathrm{CN})$ at the SANS-I instrument [18] of the SINQ facility at PSI and with very cold neutrons (VCN) and UCN at the PF-2 instrument [19] of the ILL. The total cross sections have been measured using the transmission technique 


$$
\sigma=\frac{\ln \left(I_{0} / I\right)}{N d}
$$

where $I_{0}$ and $I$ are the transmitted intensities for the target cell empty and full, $N$ is the number density, and $d$ the thickness of the target. Neutron energy $(E)$ measurements have been made using velocity selection and time of flight; $E$ was converted to in-medium kinetic energy $E^{\prime}=E-U$ (see, e.g., [1]), using the Fermi potential $U=\frac{2 \pi \hbar^{2}}{m_{n}} N b_{\text {coh }}=$ $104 \mathrm{neV}$ for solid deuterium with the density $N=3.0 \times$ $10^{22} \mathrm{~cm}^{-3}$ [20] and the bound coherent scattering length $b_{\text {coh }}=2 \times 6.67 \mathrm{fm} \mathrm{[21].}$

The ${ }^{2} \mathrm{H}_{2}$ had a purity of at least $99.95 \%$. It was prepared with a high orthoconcentration of $c_{o}=(98.6 \pm 0.4) \%$ using OXISORB ${ }^{\circledR}[22]$ at temperatures around the triple point $(18.7 \mathrm{~K})$ as a catalyst. The $c_{o}$ value was measured in situ with Raman spectroscopy of the solid deuterium [14]. The para- ${ }^{2} \mathrm{H}_{2}$ fraction contributes about $1 / v \times(1-$ $\left.c_{o}\right) \times 220 \mathrm{bm} / \mathrm{s}$ to the measured cross sections (estimated using [23]). The maximum possible $H$ contamination $c_{H}=0.0005$ contributes $c_{H} \times 82 \mathrm{~b}$ incoherent scattering and $1 / v \times c_{H} \times 730 \mathrm{bm} / \mathrm{s}$ absorption cross section. These contributions are comparable to the absorption on deuterons $(1 / v \times 1.1 \mathrm{bm} / \mathrm{s})$ and close to negligible (see measured cross sections below).

The measurements have been performed with the deuterium target at $(18.2 \pm 0.1)$ and $(5.3 \pm 0.1) \mathrm{K}$, respectively. The solid ${ }^{2} \mathrm{H}_{2}$ densities $\rho_{18 \mathrm{~K}}^{-1}=(20.38 \pm$ $0.02) \mathrm{cm}^{3} / \mathrm{mol}$ and $\rho_{5 \mathrm{~K}}^{-1}=(19.93 \pm 0.01) \mathrm{cm}^{3} / \mathrm{mol}$ were taken from [20]. Solid angle corrections are small and range between $10^{-2}$ and $10^{-3}$. A major experimental uncertainty is the actual target thickness $d$, due to using thin windows, which bulge. We used windows machined from aluminum alloy (AlMg3/AA5754) rods to thicknesses of $150-200 \mu \mathrm{m}$. The windows must withstand, typically, a pressure of $1 \mathrm{~atm}$ against vacuum. The target thickness for flat windows is $d=10 \mathrm{~mm}$. In the analysis, effective target thicknesses were used; $d=10 \mathrm{~mm}$ for the $\mathrm{CN}$ and VCN data for one set of windows and $d=$ $11.1 \mathrm{~mm}$ for the UCN data taken with another set of windows (These values are in agreement with our findings during the measurements of liquid deuterium [15]). The resulting overlap of the data sets is good, however, we estimate a common systematic uncertainty of 5\%-10\% for the target thickness of the three individual data sets and the corresponding cross section values.

The results of the measurements for a target at $18 \mathrm{~K}$ are displayed in Fig. 1 together with those from the only other solid ${ }^{2} \mathrm{H}_{2}$ cross section measurement made to date, for neutrons in the energy range 1.4 to $80 \mathrm{meV}$ and at a temperature of $17 \mathrm{~K}$, by Seiffert [24]. The two sets of results are in good agreement in the overlapping energy

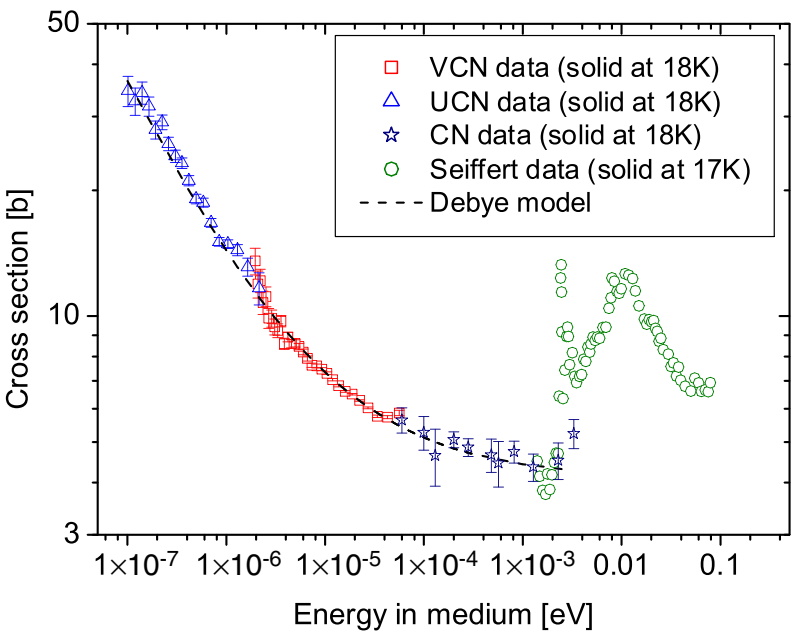

FIG. 1 (color online). Measured cross sections per deuterium molecule. Included in the plot is the data of [24]. The expected cross section is dominated by thermal up scattering and calculated using the Debye model.

region below about $2 \mathrm{meV}$. Above $2 \mathrm{meV}$ coherent (Bragg) scattering is possible (cutoff wavelength $0.61 \mathrm{~nm}[24,25]$ ) but depends on details of the particular target crystal; in addition Bragg peaks are suppressed in our experiment due to the poor velocity resolution (about 10\%) in the smallangle neutron-scattering technique measurement. Also included in Fig. 1 is the calculated cross section taking into account thermal up scattering and the minor contributions of up scattering in para $-{ }^{2} \mathrm{H}_{2}$ and absorption (These contributions are maximal at the lowest neutron energies and there amount to an increase in cross section by $2.5 \%$ ). The thermal up scattering contribution has been calculated using a simple Debye model for solid deuterium, similar to that used by, e.g., [5,23].

The normalized phonon density of states is taken to be $Z(\omega)=3 \omega^{2} / \omega_{D}^{3}$, where the effective maximum phonon frequency $\omega_{D}=k_{B} \theta_{D} / \hbar$ and the Debye temperature, $\theta_{D}=110 \mathrm{~K}$ [26]. At the low temperatures considered here, the orthodeuterium molecule is in its ground state and spherically symmetric; it may be considered as a single particle with a bound scattering length $2 b j_{0}\left(\frac{Q a}{2}\right)$ [25] in which $b^{2}=b_{\text {coh }}^{2}+5 / 8 b_{\text {inc }}^{2}$ (following from the statistical weights of the nuclear spin configurations $S=0,2$ of ortho- ${ }^{2} \mathrm{H}_{2}$; see also [27-29]), the spherical Bessel function $j_{0}$, the distance of the deuterons in the molecule, $a=$ $0.74 \AA$, and the momentum transfer $Q=\left|\mathbf{k}-\mathbf{k}^{\prime}\right|$, where $\mathbf{k}$ and $\mathbf{k}^{\prime}$ are the wave vectors of the initial state and the final state of the neutron, respectively. Following [23] we use results for a cubic lattice, rather than the actual hcp structure [25], so that the single phonon scattering cross section is written as

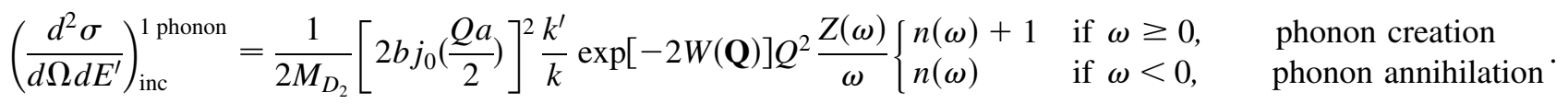

The population number $n(\omega)=\left(\exp \left(\frac{\hbar \omega}{k_{B} T}\right)-1\right)^{-1}$ of phonons with the frequency $\omega=\hbar Q^{2} /\left(2 M_{n}\right)$ is given by Bose- 
Einstein statistics, while the Debye-Waller factor, $\exp [-W(\mathbf{Q})]$, with $W(\mathbf{Q})=\frac{1}{6} Q^{2}\left\langle\mathbf{u}^{2}\right\rangle$ [30] and $\left\langle\mathbf{u}^{2}\right\rangle=$ $0.25 \AA^{2}$ [25] being the mean squared displacement of the nuclei, describes the zero phonon expansion of the cubic lattice. The up scattering cross section is obtained by integrating Eq. (2) over all final energies starting from the initial neutron energy.

The good agreement between the measured and calculated cross sections for VCN and UCN is a direct validation for the scattering model, which could only be inferred indirectly from earlier measurements [7,9]. A direct consequence of this agreement is that the deuterium crystals produced at $18 \mathrm{~K}$ would seem to be close to "ideal."

Figure 2 shows the results for three different sets of measurements with deuterium at $5 \mathrm{~K}$, (i) with very slow cooling (less than $1 \mathrm{~K}$ per hour), (ii) after temperature cycling between 5 and $10 \mathrm{~K}$, and (iii) after temperature cycling between 5 and $18 \mathrm{~K}$ : In contrast to the measurements at $18 \mathrm{~K}$, the model does not describe the measured data and the way the crystal is treated dominates the results to the point where applying Eq. (1) is no longer meaningful. The probable cause is scattering by crystal imperfections introduced by cooling from 18 to $5 \mathrm{~K}$ and exacerbated by the temperature cycling.

The first evidence for this explanation is that the change in molar volume over the temperature range 5 to $10 \mathrm{~K}$ is $0.2 \%$ and over the range 5 to $18 \mathrm{~K}$ is $2 \%$ [20]: Solidifying at $18 \mathrm{~K}$ and then cooling to $5 \mathrm{~K}$ cannot avoid introducing strain, and temperature cycling to $18 \mathrm{~K}$ involves a strain cycle a factor of 10 larger than for cycles over the temperature range 5 to $10 \mathrm{~K}$. Support for this explanation comes from the similar behavior of the light transmission of solid ${ }^{2} \mathrm{H}_{2}$ [14] under similar thermal treatment. Crystals produced at $18 \mathrm{~K}$ were optically transparent with few visible imperfections, while those cooled from 18 to $5 \mathrm{~K}$

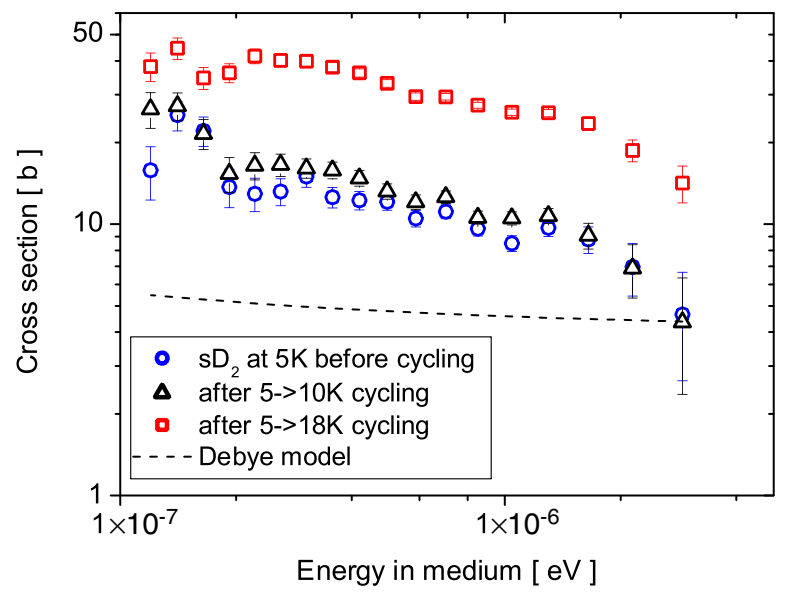

FIG. 2 (color online). Measured cross sections per deuterium molecule for UCN energies. The data sets correspond to the differently treated target crystals measured at $5 \mathrm{~K}$, either after careful cooldown only, or after thermal cycles between 5 and $10 \mathrm{~K}$ or between 5 and $18 \mathrm{~K}$. The expected cross section for the ideal crystal can obviously not describe the measured data. were always cloudy, irrespective of the amount of care taken. Thermal cycling between 5 and $18 \mathrm{~K}$ results in a reduction of the light transmission by a factor of $10 \mathrm{com}-$ pared to a $10 \%$ reduction in the case of cycles between 5 and $10 \mathrm{~K}$; this is illustrated in Fig. 3 where the measured light transmission through a solid ${ }^{2} \mathrm{H}_{2}$ sample over the period of a sequence of thermal cycles similar to those used in the neutron experiment is plotted. Starting with a crystal at $5 \mathrm{~K}$ prepared using the most careful procedures [similar to that used for case (i) of the neutron measurements], regions $\mathrm{A}$ and $\mathrm{C}$ are with the sample held at a constant temperature of $5 \mathrm{~K}$, region $\mathrm{B}$ is a sequence of 7 temperature cycles between 5 and $10 \mathrm{~K}$, and region $\mathrm{D}$ a sequence of 7 temperature cycles between 5 and $18 \mathrm{~K}$. For the larger temperature cycles some recovery of the transmission can be seen in each cycle but the trend is for a fast reduction. Sequences with 10 and more cycles showed almost the same relative transmission losses, indicating that the full reduction of transmission loss is reached after 7 or so cycles.

Although one needs to be cautious about drawing general conclusions from results obtained from crystals grown in a specific target cell, the evidence is that the lowest values measured at $5 \mathrm{~K}$ probably set the limit for what can be achieved with crystals prepared from the liquid phase. The best chance for producing ideal solid deuterium at $5 \mathrm{~K}$ will be freezing directly from the gas phase to a temperature below $10 \mathrm{~K}$; this was not possible with the target cell used in this experiment. Another important practical result for UCN source design from the measurements is that the solid ${ }^{2} \mathrm{H}_{2}$ should be kept below $10 \mathrm{~K}$ at all times, otherwise a systematic deterioration of the performance of the converter can result (or new ${ }^{2} \mathrm{H}_{2}$ crystals will have to be produced regularly if the performance of the source is to be maintained). In the case of the UCN source at PSI $[10,11]$, the UCN production will be in pulses of several

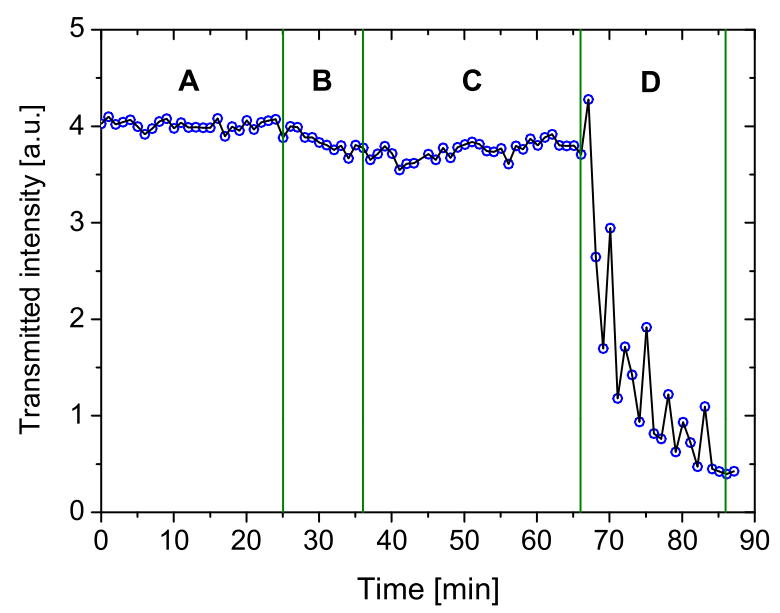

FIG. 3 (color online). The variation of the intensity of $488 \mathrm{~nm}$ light from an argon-ion laser transmitted by solid ${ }^{2} \mathrm{H}_{2}$ crystals subjected to a sequence of thermal cycles. See text for explanations and [14] for details of the measurement method. 
seconds duration at $1 \%$ duty cycle. During an $8 \mathrm{~s}$ production pulse the temperature of the ${ }^{2} \mathrm{H}_{2}$ is calculated to increase from 5 to $9 \mathrm{~K}$. It is recooled to $5 \mathrm{~K}$ over the next $800 \mathrm{~s}$.

This work was performed at the Swiss Spallation Neutron Source SINQ; Paul Scherrer Institut, Villigen, Switzerland; and at the Institute Laue-Langevin, Grenoble, France. We are grateful to various technical support services, whose outstanding efforts have made these experiments possible. We especially acknowledge the help of W. Arrigoni, T. Brenner, K. Kohlik, and P. Schurter. We gratefully acknowledge the contribution of M. Giersch for the Raman spectroscopy and during beam time. We would also like to thank M. Hino and M. Utsuro for providing the VCN chopper at the ILL. Helpful discussions with C.-Y. Liu are gratefully acknowledged. This work is supported by the Swiss National Science Foundation (Grant No. 2100-067840).

*Also at Institute for Technical Chemistry, ETH Zürich, Switzerland.

${ }^{\dagger}$ Also at Physik-Institut, University of Zürich, Switzerland. ${ }^{\ddagger}$ Corresponding author.

Electronic address: klaus.kirch@psi.ch

${ }^{\S}$ Electronic address: http://ucn.web.psi.ch

"Present address: Paul Scherrer Institut, CH-5232 Villigen PSI, Switzerland, and Stefan Meyer Institut für subatomare Physik, Austrian Academy of Sciences, Vienna, Austria.

${ }^{\mathbb{I}}$ Also at Paul Scherrer Institut, CH-5232 Villigen PSI, Switzerland.

[1] V. K. Ignatovich, Ultracold Neutrons (Clarendon, Oxford, 1990).

[2] R. Golub, D. J. Richardson, and S. K. Lamoreaux, UltraCold Neutrons (Adam Hilger, Bristol, 1991).

[3] I. S. Altarev et al., Phys. Lett. 80A, 413 (1980).

[4] R. Golub and K. Böning, Z. Phys. B 51, 95 (1983).

[5] Z.-Ch. Yu, S. S. Malik, and R. Golub, Z. Phys. B 62, 137 (1986).

[6] A. Serebrov et al., Nucl. Instrum. Methods Phys. Res., Sect. A 440, 658 (2000).
[7] C. L. Morris et al., Phys. Rev. Lett. 89, 272501 (2002).

[8] A. Saunders et al., Phys. Lett. B 593, 55 (2004).

[9] F. Atchison et al., Phys. Rev. C 71, 054601 (2005).

[10] At PSI we are presently setting up a high intensity source of UCN using solid deuterium (http://ucn.web.psi.ch/). Other efforts, known to us, are underway at Los Alamos National Laboratory, the Technical University Munich, the North Carolina State University, and at Mainz University.

[11] F. Atchison et al., in The PSI UCN Source, Proceedings of the International Collaboration on Advanced Neutron Sources, ICANS-XVII, Santa Fe, 2005 (to be published).

[12] Yu. N. Pokotilovski, Nucl. Instrum. Methods Phys. Res., Sect. A 356, 412 (1995).

[13] A. P. Serebrov et al., JETP Lett. 66, 802 (1997).

[14] K. Bodek et al., Nucl. Instrum. Methods Phys. Res., Sect. A 533, 491 (2004).

[15] F. Atchison et al., Phys. Rev. Lett. 94, 212502 (2005).

[16] M. Kasprzak, Diploma thesis, Jagiellonian University, Krakow, 2004; nucl-ex/0407022.

[17] F. Atchison et al., in Investigation of Solid Deuterium for UCN Sources, Proceedings of the 17th Meeting of the International Collaboration on Advanced Neutron Sources, ICANS-XVII, Santa Fe, 2005 (to be published).

[18] J. Kohlbrecher and W. Wagner, J. Appl. Crystallogr. 33, 804 (2000).

[19] A. Steyerl et al., Phys. Lett. A 116, 347 (1986).

[20] P.C. Souers, Hydrogen Properties for Fusion Energy (University of California, Berkeley, 1986).

[21] V.F. Sears, Neutron News 3 No. 3, 26 (1992).

[22] OXISORB ${ }^{\circledR}$ is obtained from the MESSER company.

[23] C.-Y. Liu, A. R. Young, and S. K. Lamoreaux, Phys. Rev. B 62, R3581 (2000).

[24] W.-D. Seiffert, Euratom Report NO. EUR 4455 d, 1970.

[25] M. Nielsen and H.B. Moller, Phys. Rev. B 3, 4383 (1971).

[26] I. F. Silvera, Rev. Mod. Phys. 52, 393 (1980).

[27] J. Schwinger and E. Teller, Phys. Rev. 52, 286 (1937).

[28] M. Hamermesh and J. Schwinger, Phys. Rev. 69, 145 (1946).

[29] J. A. Young and J. U. Koppel, Phys. Rev. 135, A603 (1964).

[30] S.W. Lovesey, Theory of Neutron Scattering from Condensed Matter (Clarendon, Oxford, 1984). 